\title{
From the time-wise to space-wise GOCE observables
}

\author{
M. Reguzzoni \\ DIIAR, Sez. Rilevamento, Politecnico di Milano, Via Valleggio, 11 - 22100 Como, Italy
}

\begin{abstract}
The observables of the GOCE three-axis gradiometer are taken in time, along the orbit and, in the timewise proposal, filtered to stay in the measurement bandwidth, i.e. in the frequency interval between 0.005 and $0.1 \mathrm{~Hz}$. As a consequence, the resulting "observable" is a convolution of the original data stream with a time-wise weighting kernel. In other words, we cannot assume that the observations are point-wise evaluations of any function and so, in a spacewise approach, any averaging or interpolating operation to obtain gridded spatial data has little sense.

The problem is therefore to model correctly the observational functionals, including the correlation along the orbit. This can be done by exploiting the Wiener filter theory, using the prior knowledge of a geopotential model and the power spectral density (PSD) of the measurement error.

A numerical simulation from the EGM96 model (degrees between 50 and 300) is performed, showing that the along track Wiener filter produces $T_{r r}$ spatialized observables with an error standard deviation of the order of $5 \mathrm{mE}$. A covariance function of the estimation error is also provided by the Wiener filter theory. The use of these filtered observables in a space-wise approach allows for the reconstruction of the gravity field in terms of spherical harmonics up to degree 200.
\end{abstract}

Key words. Satellite gradiometry, space-wise approach, measurement bandwidth, Wiener filter

\section{Introduction}

The satellite mission GOCE will provide a new type of space observations, the so-called gradiometric data, i.e. abstracting, the second derivatives of the gravitational potential $T$ at given points along the orbit.

In theory these observations are spatial data and we can think of applying a space-wise approach to retrieve the har-

Correspondence to: M. Reguzzoni

(mirko@geomatica.ing.unico.it) monic coefficients of the Earth potential; basically this approach consists in homogenizing the gradiometric data in terms of spatial proximity, for example estimating the second radial derivatives $T_{r r}$ (and maybe $T_{\lambda \lambda}$ ) on grid points from a "local" set of observed data $\left(T_{x x}, T_{y y}, T_{z z}\right)$, and then applying on this smaller regular data-set a fast collocation algorithm (Sansò and Tscherning, 2001) or a simple integration method to determine a global geopotential model. In this way the numerical complexity is reduced and, in addition, one hopes to control the time-wise biases by grouping data which are "close in space" but "far in time".

As a matter of fact the three-axis gradiometer, which will be on board the GOCE satellite, contains such a strongly time-wise correlated noise that it does not allow for the direct use of the GOCE measurements in a space-wise approach.

The paper deals with the problem of the treatment of the coloured noise by introducing a proper filtering method. This is a typical time-wise procedure, but in this framework it is considered as an intermediate step, a preprocessing from the time-wise to space-wise observables, or at least to a more spatialized data than the original ones. In particular a Wiener filter, exploiting the correlation along the orbit, seems to be suitable to our purpose, while a simple box shape filter does not give acceptable results.

Naturally this proposal is not the only one possible; something similar is done in the time-wise approach, though by slightly different stochastic models (Koop et al., 2000; Schuh, 2000).

\section{The measurement bandwidth filter}

The power spectral density (PSD) of the measurement error of the GOCE gradiometer shows high values both at low and high frequencies, such that the optimal measurement frequencies, the so-called measurement bandwidth (M.B.), are in the interval 0.005 to $0.1 \mathrm{~Hz}$ (see Fig. 1). 


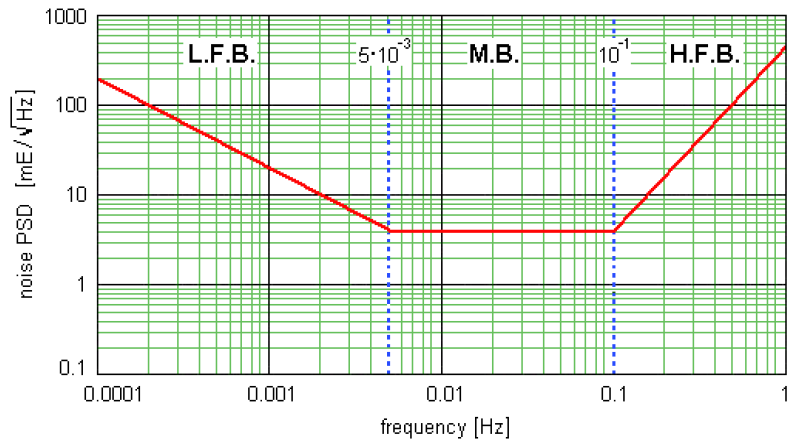

Fig. 1. The measurement error PSD.

So the first and simplest idea is to filter the GOCE data "to stay" in the measurement bandwidth by means of a box shape filter. In the frequency domain, it can be written as

$R(p)= \begin{cases}1 & p_{\ell}<p<p_{h} \\ 0 & \text { otherwise }\end{cases}$

or in the time domain, in terms of weighting kernel,

$K_{R}(t) \propto A \cdot \operatorname{sinc}\left(2 \pi p_{h} t\right)-a \cdot \operatorname{sinc}\left(2 \pi p_{\ell} t\right)$

where $A / a=p_{h} / p_{\ell}, p_{\ell}=0.005 \mathrm{~Hz}$ and $p_{h}=0.1 \mathrm{~Hz}$, i.e. the low and high cut frequencies of the gradiometer measurement bandwidth. The rectangular filter and its weighting kernel are shown in Fig. 2 and Fig. 3.

Numerical experiments have been performed to determine which harmonic components are affected by the signal "loss" due to the M.B. filter (Albertella et al., 2002a). As a matter of fact what happens for the different degrees $\ell$ is that:

- for $\ell<25$, all the information is completely contained in the low frequency band (L.F.B.) (see Fig. 1) and so it is definitively lost;

- for $25<\ell<300$, the information is spread between L.F.B. and M.B.; in any case, up to degree 300 , there is no relevant signal in the high frequency band (H.F.B.) and hence the upper cut frequency of the filter is not even approached.

However, to evaluate the performance of this simple filter we have simulated about $4 \cdot 10^{6} T_{r r}$ data (corresponding to 50 days of observations) based on the EGM96 model from degree 50 up to degree 300 . The satellite orbit was supposed to be circular and polar, at an altitude of $250 \mathrm{~km}$. The noise to be added to the simulated signal has been computed according to the spectrum shown in Fig. 1. Of course the spectrum of the simulated noise is discrete and the minimum frequency is taken as the inverse of the number of data.

Essentially the GOCE observations can be written as

$y_{0}(t)=\boldsymbol{s}(t)+\boldsymbol{v}(t)$

where $s(t)$ is the vector of the second radial derivatives along the orbit and $\boldsymbol{v}(t)$ is the vector of the observation noise. The behaviours of the signal and the noise are shown in Fig. 4 and

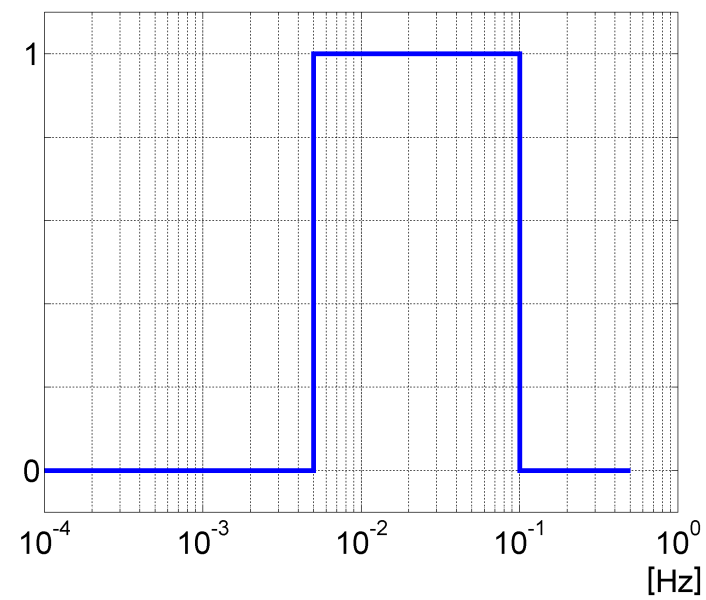

Fig. 2. M.B. filter.

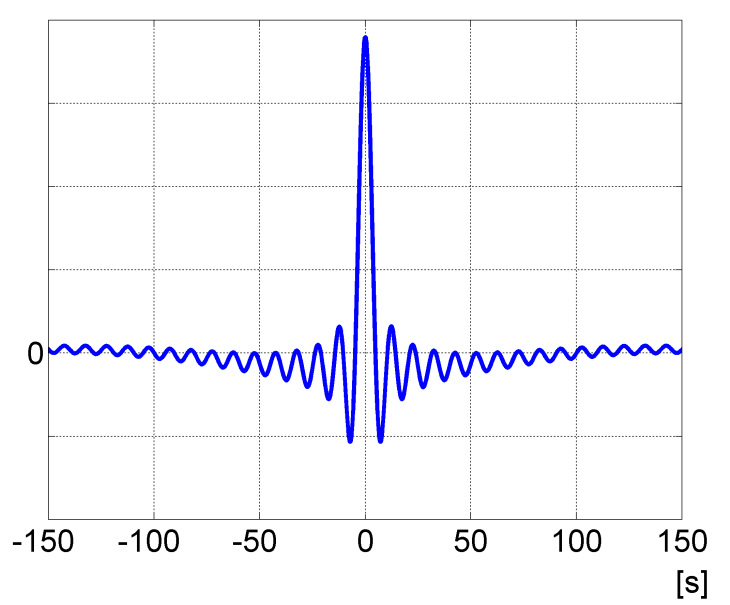

Fig. 3. M.B. weighting kernel.

Fig. 5, respectively. The visual effect that makes the signal look like a "noise" and viceversa is due to the fact that the true noise includes much larger wavelengths than the signal.

Taking into account the fact that in our simulation the signal standard deviation is of the same order of the noise standard deviation (about $85 \mathrm{mE}$ ), it is clear that the point-wise information coming from Eq. (3) is totally insufficient to estimate the "real" signal and then derive the gravity field.

If we apply the M.B. filter to the flow of the noisy $T_{r r}$ observations, the estimation error decreases down to $45 \mathrm{mE}$, which is half the original noise, but still too high to apply any point-wise analysis, numerically proving the poor performance of the rectangular filter (1). Note that the result does not change significantly if we smooth the edges of the filter in order to reduce the unwanted oscillations in the weighting kernel.

If we want to squeeze all the information from the observations, we have to consider also the signal and the noise correlation in our filtering procedure. This can be done by exploiting the Wiener filter theory. 


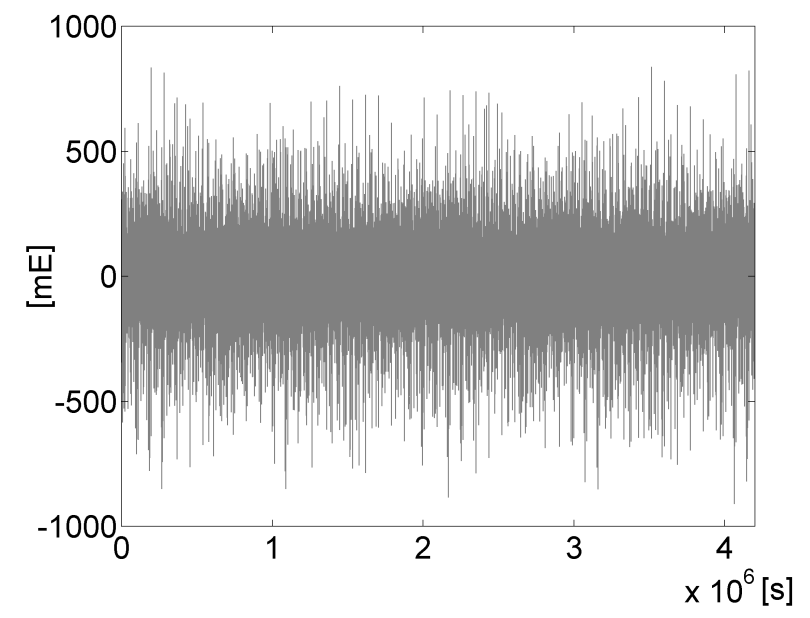

Fig. 4. $T_{r r}$ simulated signal.

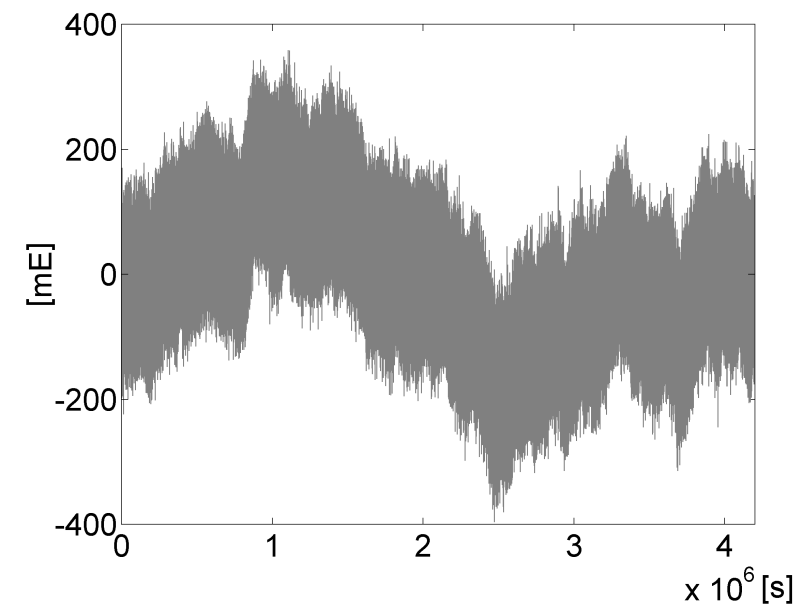

Fig. 5. Simulated noise.

\section{The Wiener filter}

Starting from Eq. (3), we make the basic hypothesis that $s(t)$ and $\boldsymbol{v}(t)$ are realizations of stationary processes, independent from one another. In this way we can estimate their covariance functions, $C_{s s}(\tau)$ and $C_{\nu v}(\tau)$, from one realization only and, denoting with $F\{\cdot\}$ the Fourier transform operator, we can compute the signal and the noise power spectra

$$
\left\{\begin{array}{l}
S_{s}(p)=F\left\{C_{s s}(\tau)\right\} \\
S_{v}(p)=F\left\{C_{v v}(\tau)\right\}
\end{array} .\right.
$$

If we minimize the mean square estimation error in the time domain, we obtain the filtered estimates of the signal

$\hat{\boldsymbol{s}}(t)=\mathbf{C}_{s s}\left(\mathbf{C}_{s s}+\mathbf{C}_{v v}\right)^{-1} \boldsymbol{y}_{\mathbf{0}}(t)$.

From the numerical point of view, it is more convenient to work in the frequency domain, where we get

$\hat{\boldsymbol{s}}(t)=F^{-1}\left\{\frac{S_{S}(p)}{S_{S}(p)+S_{v}(p)} \tilde{\boldsymbol{y}}_{0}(p)\right\} ;$

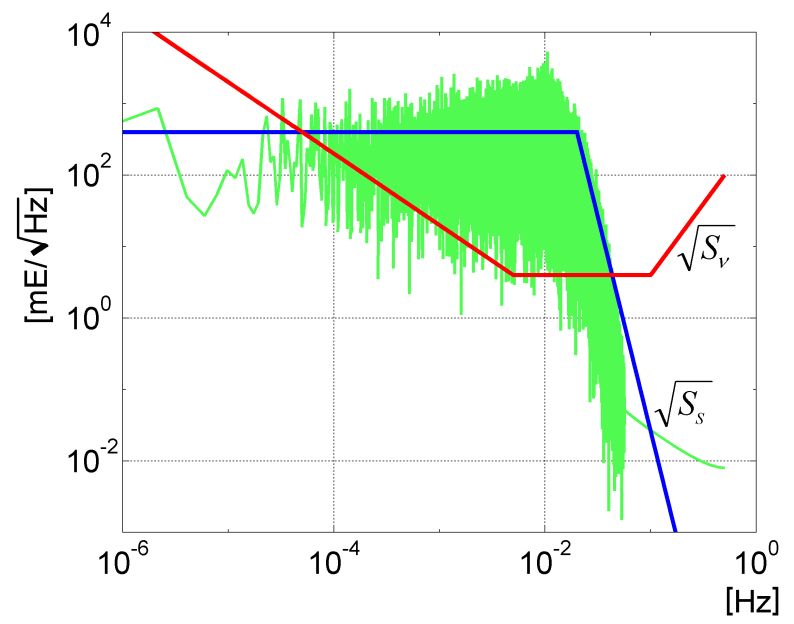

Fig. 6. Empirical and analytical signal spectra and analytical noise spectrum.

here $\tilde{y}_{\mathbf{0}}(p)=F\left\{\boldsymbol{y}_{\mathbf{0}}(t)\right\}$ and

$W(p)=\frac{S_{S}(p)}{S_{S}(p)+S_{v}(p)}$

is the so-called Wiener filter (Papoulis, 1984).

This stochastic approach enables us to estimate also the covariance structure of the estimation error and hence to have a "measure" of the performance of the filtering procedure; in fact, defining

$\hat{\boldsymbol{e}}(t)=\hat{\boldsymbol{s}}(t)-\boldsymbol{s}(t)$

the error covariance function can be written as

$C_{\hat{e} \hat{e}}(\tau)=F^{-1}\left\{\frac{S_{S}(p) \cdot S_{v}(p)}{S_{S}(p)+S_{v}(p)}\right\}$.

The first step in order to build the Wiener filter is to determine the spectra.

The noise spectrum depends on the GOCE gradiometer, it is displayed in Fig. 1 and it is considered as "known" at least for the purpose of the Wiener filter. Its analytic expression can be written as

$\sqrt{S_{v}(p)}=\left\{\begin{array}{ll}0.02 \cdot p^{-1} & p<p_{\ell} \\ 4 & p_{\ell} \leq p<p_{h} \\ 400 \cdot p^{2} & p \geq p_{h}\end{array} \quad[\mathrm{mE} / \sqrt{\mathrm{Hz}}]\right.$.

As for the signal, using the same $T_{r r}$ simulated data of Fig. 4, we can compute the empirical spectrum, shown in Fig. 6. Note that this signal spectrum depends on which geopotential model has been chosen as prior knowledge, in this case EGM96, though this dependence is quite weak (Albertella et al., 2002b). For this reason, the empirical spectrum has been interpolated using the following analytic formula:

$\sqrt{S_{S}(p)}=\left\{\begin{array}{ll}400 & p<p_{c} \\ 256 \cdot 10^{-10} \cdot p^{-6} & p \geq p_{c}\end{array}[\mathrm{mE} / \sqrt{\mathrm{Hz}}]\right.$

where $p_{c}=0.02 \mathrm{~Hz}$. 


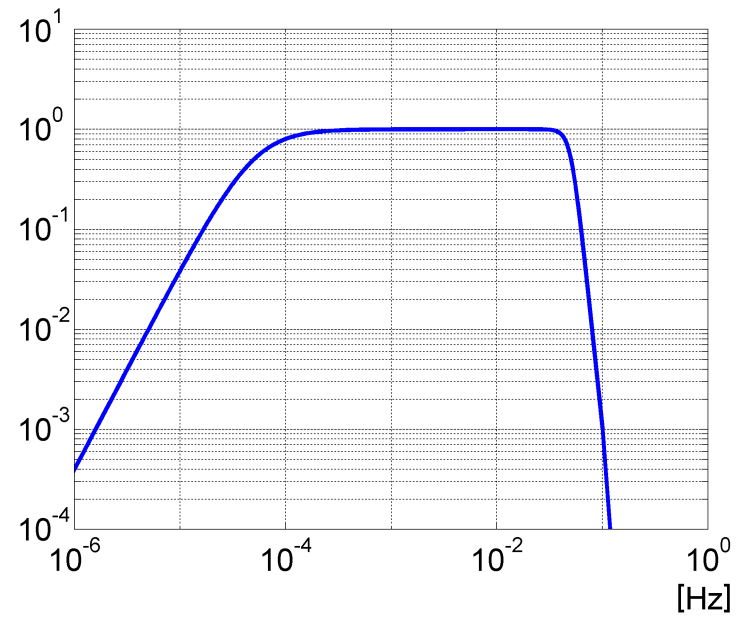

Fig. 7. Wiener filter.

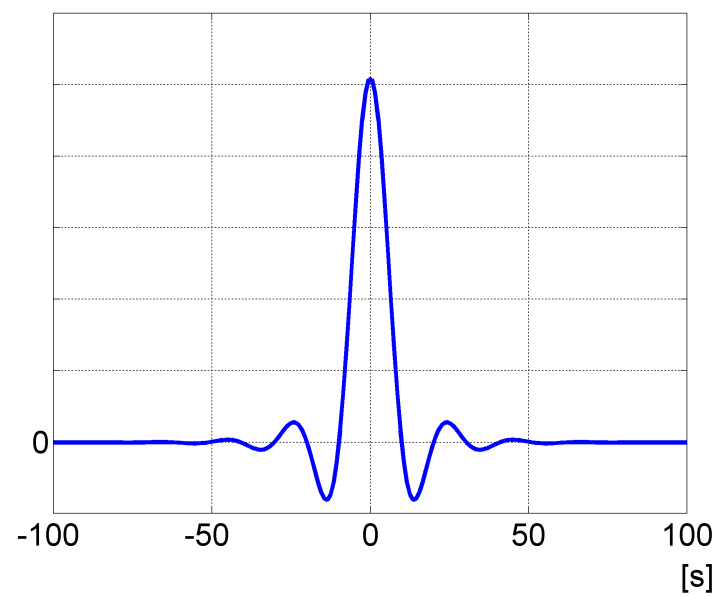

Fig. 8. Wiener filter weighting kernel.

The analytic models are also shown in Fig. 6. By putting them into Eq. (7), we can explicitly obtain the Wiener filter (see Fig. 7) and derive its related weighting kernel in the time domain by back-transforming (see Fig. 8).

Some comments are due about the characteristics of the Wiener filter. As the signal spectrum is above the noise spectrum also below the cut frequency of $5 \mathrm{mHz}$ (see Fig. 6), the width of the Wiener filter band-pass is much larger than the one of the M.B. filter in the low frequency, reducing the signal loss in this part of the spectrum; in fact, as already written before, the information in L.F.B. does not come only from the low degrees, which we assume to be known, but also from the medium-high degree coefficients, whose determination is the main purpose of a space mission like GOCE. Furthermore, comparing the weighting kernels in Fig. 3 and Fig. 8, it is clear that the Wiener filter involves less data in the estimate of the single $T_{r r}$ along the orbit and as a consequence it spreads the point-wise information onto a smaller time lag. This is useful in the subsequent space-wise treatment in order to retrieve the harmonic coefficients.

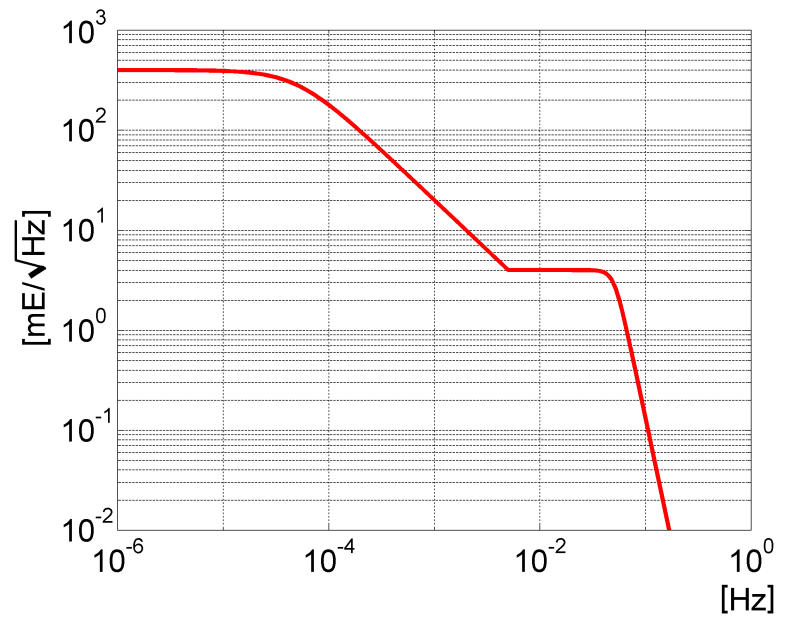

Fig. 9. PSD of the estimation error (Wiener filter).

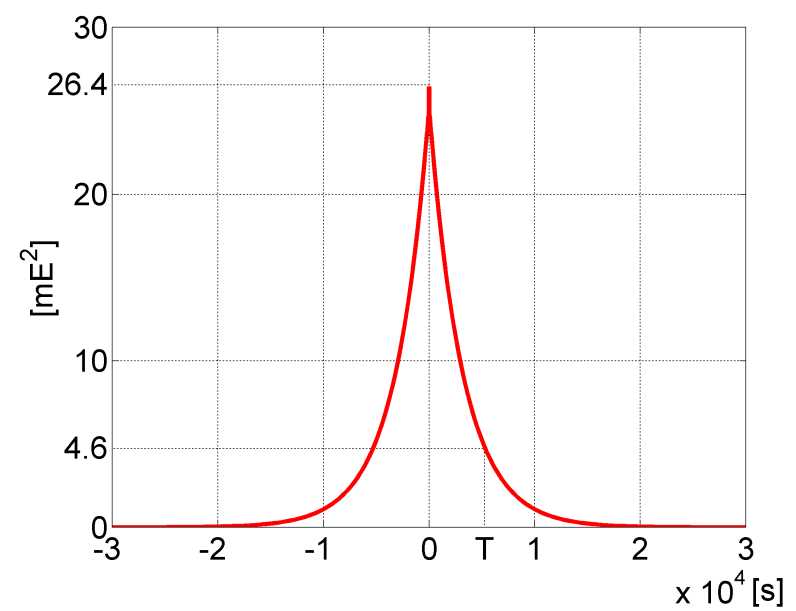

Fig. 10. Covariance function of the estimation error (Wiener filter). $\mathrm{T}=$ orbital period.

Multiplying the Fourier transform of the observations by the Wiener filter and back-transforming, as in Eq. (6), we obtain the filtered signal and then determine its empirical error standard deviation, that is equal to $5.1 \mathrm{mE}$, much smaller than that one calculated for the M.B. filter. Moreover, using Eq. (9) it is also possible to compute the theoretical PSD and the covariance function of the estimation error, shown in Fig. 9 and Fig. 10. As one can see, the theoretical value of the error standard deviation is practically identical to the empirical one.

Note that the covariance function in Fig. 10 has a strongly correlated tail; after one orbital period the correlation is still the $20 \%$ of the variance; this is typical in a Wiener filter approach. Anyway, this covariance function opens the opportunity of realizing a crossover check on $T_{r r}$ at the level of $6.5 \mathrm{mE}$, which could be used in a calibration procedure.

As a final remark, we underline that we have worked here with $T_{r r}$ data only, because they are the easiest to be used in simulations, but the Wiener filter approach can be ap- 


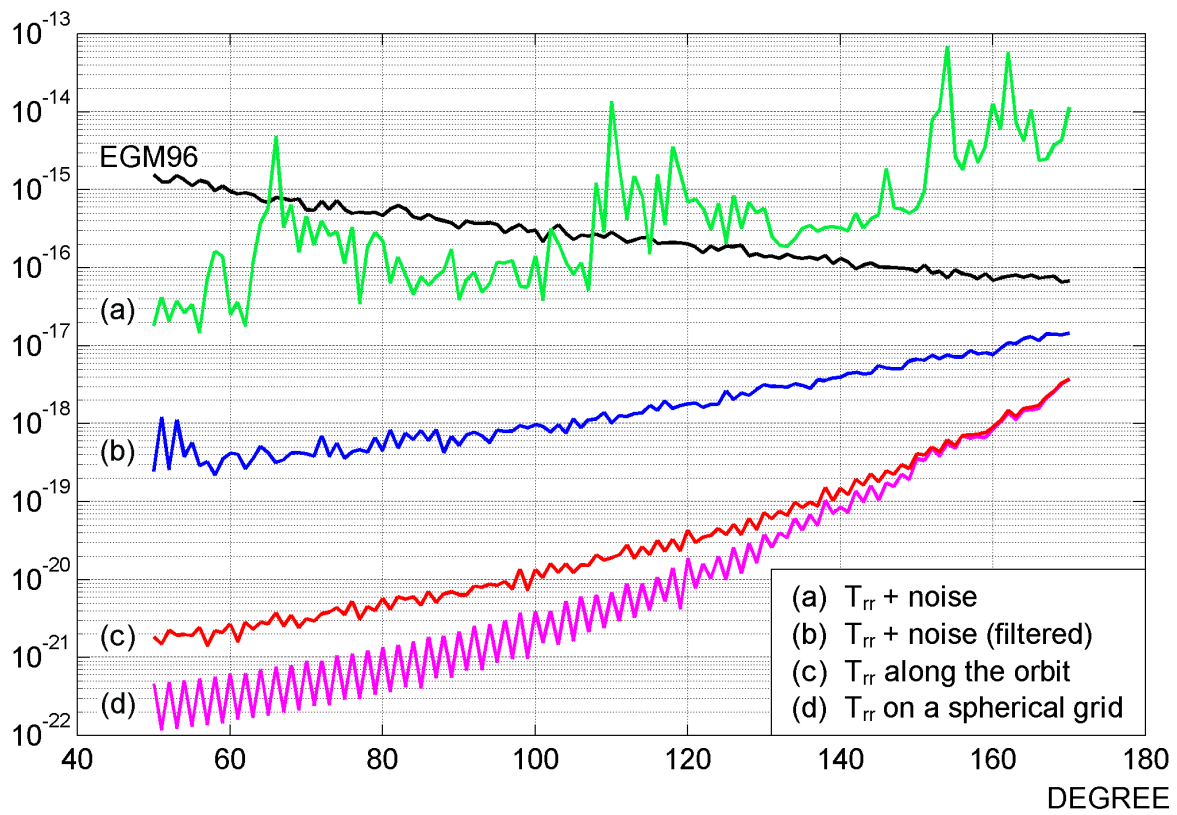

Fig. 11. Error degree variances of the estimated models.

plied as well to the other components of the gravity tensor, for instance by means of a multiple input single output filter (MISO) (Albertella et al., 2002b). In this way we expect to consistently increase the quality of our results.

\section{First results on the gravity field recovery}

The Wiener filtered observations can be considered a product by themselves, but in the GOCE framework they represent an input for the gravity field determination procedure; in particular we are interested in a space-wise approach.

In a strict sense, the filtered observations are not "local" measurements, i.e. point-wise evaluations of any function; in fact they are spread onto a time lag due to the convolution with the filtering kernel. As a consequence, in a space-wise approach, any averaging or interpolating procedure to obtain gridded spatial data from such "observations" has little sense.

Anyway the Wiener filter kernel is quite narrow and the standard deviation of the estimation error is much smaller than that one of the signal. So we can assume that the filtered data have point-wise meaningful information.

A numerical simulation has been performed, with the same circular and polar orbit, starting from EGM96 (from degree 50 to 300 ); using a space-wise approach, in terms of leastsquares interpolation followed by spherical harmonic integration, the global gravity field has been estimated from:

- $T_{r r}$ directly generated on a spherical grid of $1^{\circ} \times 1^{\circ}$ : the estimation error depends only on the harmonic integration procedure;
- $T_{r r}$ simulated along the orbit, without noise: the additional error depends on the interpolation procedure and it could be considered as negligible;

- $T_{r r}$ simulated along the orbit, with noise: the estimated model is too inaccurate;

- Wiener filtered $T_{r r}$.

The results of this simulation are shown in Fig. 11, in terms of error degree variances of the estimated coefficients compared with the degree variances of the reference model. In order to bound the aliasing and control the discretization error, the harmonic integration extends just below degree 180 , that is the theoretical limit for a $1^{\circ} \times 1^{\circ}$ grid (Rummel et al., 1993); however the use of the Wiener filtered observables seems, in the context of this simulation, to allow for the reconstruction of the gravity field up to degree 200 with sufficient accuracy. The effects of iterating the procedure to obtain an improved estimate of the potential coefficients are under study.

\section{Conclusions}

In conclusion we can make the following remarks:

- the along track Wiener filter produces "spatialized" observables, with low-value (but long-tailed) estimation errors; the magnitude of such an error (about $5.1 \mathrm{mE}$ ) is very close to the threshold defined for the GOCE mission even using $T_{r r}$ data only; this result, valuable per se, can be improved by considering a joint filter for all the components; 
- the filtered point-wise data can be locally interpolated to produce a quite reasonable geographic grid of pseudoobservations, which can then be worked out in a quadrature approach (Migliaccio and Sansò, 1989) or by some more sophisticated methods (Sansò and Tscherning, 2001);

- the along orbit filtered values can also be used to test, at crossovers, the absence of biases in the measurements.

So, though still very crude, the Wiener filter seems to provide a required step to arrive at a full space-wise data analysis.

\section{References}

Albertella, A., Migliaccio, F., Reguzzoni, M., and Sansò, F.: "Spacewise approach and measurement bandwidth in satellite gradiometry", Bollettino di Geodesia e Scienze Affini, 3, 179189, 2002a.

Albertella, A., Migliaccio, F., Reguzzoni, M., and Sansò, F.: "Wiener filters and collocation in satellite gradiometry", Proceedings of the 5th Hotine-Marussi Symposium on Mathematical Geodesy, Matera, 17-21 June, in press, 2002b.
ESA: "Gravity Field and Steady-State Ocean Circulation Mission", ESA SP-1233 (1), 1999.

Koop, R., Visser, P., Van den Ijssel, J., and Klees, R.: "Detailed scientific data processing approach", Work Package 2 of Timewise approach, in: "From Eotvos to Milligal" Final Report, Graz, April, 2000.

Migliaccio, F., and Sansò, F.: "Data Processing for the Aristoteles Mission", Proceedings of the Italian Workshop on the European Solid-Earth Mission Aristoteles, Trevi, 30-31 May, 1989.

Papoulis, A.: "Signal analysis", McGraw-Hill, New York, 1984.

Rummel, R., Van Gelderen, M., Koop, R., Schrama, E., Sansò, F., Brovelli, M. A., Migliaccio, F., and Sacerdote, F.: "Spherical harmonic analysis of satellite gradiometry", Netherlands Geodetic Commission Publications on Geodesy, New Series, Number 39, Delft, The Netherlands, 1993.

Sansò, F., and Tscherning, C. C.: "Fast spherical collocation: a general implementation", in: International Association of Geodesy Symposia, vol. 125 "Vistas for geodesy in the new millenium", (Eds) Ádám, J. and Schwartz, K. P., Springer-Verlag, Berlin, 131-137, 2001.

Schuh, W. D.: "Scientific data processing algorithms", Work Package 3 of Time-wise approach, in: "From Eotvos to Milligal" Final Report, Graz, April, 2000. 